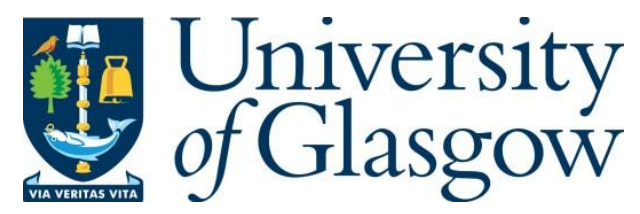

Dobbin, S., Fisher, M. and McKay, G. (2018) Management of atrial fibrillation in diabetes. Practical Diabetes, 35(1), pp. 27-31.

There may be differences between this version and the published version. You are advised to consult the publisher's version if you wish to cite from it.

This is the peer reviewed version of the following article Dobbin, S. , Fisher, M. and McKay, G. (2018) Management of atrial fibrillation in diabetes. Practical Diabetes, 35(1), pp. 27-31, which has been published in final form at http://dx.doi.org/10.1002/pdi.2155. This article may be used for non-commercial purposes in accordance with Wiley Terms and Conditions for Self-Archiving.

http://eprints.gla.ac.uk/171964/

Deposited on: 2 November 2018

Enlighten - Research publications by members of the University of Glasgow http://eprints.gla.ac.uk 


\title{
Management of atrial fibrillation in diabetes
}

\author{
Stephen Dobbin ${ }^{1}$, Miles Fisher ${ }^{2}$, Gerry McKay² \\ 1. Department of Cardiology, Golden Jubilee National Hospital, Glasgow, UK \\ 2. Department of Diabetes, Endocrinology and Clinical Pharmacology, Glasgow \\ Royal Infirmary, Glasgow, UK
}

Correspondence to: Dr Stephen Dobbin, MBChB, MRCP, Cardiology SpR, Cardiology Department, Wishaw General Hospital, Netherton Street, Wishaw ML2 0DP; email: stephen.dobbin@nhs.net

\begin{abstract}
Diabetes is an important risk factor for cardiovascular morbidity and mortality, and atrial fibrillation is linked to cardiovascular complications. The prevalence of both is increasing and they commonly co- exist with a longer duration of diabetes and poorer control putting individuals at higher risk of atrial fibrillation.

The complications of atrial fibrillation include heart failure and thromboembolism. There is an association between diabetes and atrial fibrillation and there are many theories as to the specific pathophysiology, including sharing similar precursors, but it is not clearly understood. What is understood is that the risk of developing the main consequences of atrial fibrillation can be reduced by treatment. Broadly speaking, this involves controlling the heart rhythm and/or heart rate to improve cardiac function in addition to anticoagulation. The development of risk stratification strategies and direct oral anticoagulants has led to safer anticoagulation with more individuals thought to benefit balanced against any potential risk of bleeding. The evidence base for treatment of atrial fibrillation includes many individuals with diabetes and, in this group, prevention of complications is equally as relevant. In those with diabetes and atrial fibrillation the evidence overwhelmingly supports a treatment strategy similar to that for individuals with atrial fibrillation but without diabetes. This should be done in addition to identifying and treating other cardiovascular risk factors.
\end{abstract}

\section{Introduction}

Diabetes is an important risk factor for cardiovascular morbidity and mortality, although the mechanisms are not fully understood. Similarly, atrial fibrillation (AF) is linked to cardiovascular complications, including thromboembolism, stroke and heart failure, and the prevalence of both continues to increase. ${ }^{1-4}$ 
In this article, we describe recent developments in the pathophysiology, treatment and prevention of thromboembolic complications in individuals with AF and diabetes.

\section{Pathophysiology}

There appears to be an independent association between diabetes and AF. However, it is unclear if this is a direct causal one, or if there are other indirect interactions between the conditions, and the mechanism of this link remains uncertain. ${ }^{5-8}$ Both have common precursors in the form of hypertension, atherosclerosis and obesity.

Diabetes results in defects in insulin and glucose control. This in turn can directly affect the myocardium of atria and ventricles. The underlying mechanism of AF may be related to inflammation, with biopsies of atria showing raised levels of C-reactive protein. ${ }^{9}$ Diabetes is also associated with the formation of pro-inflammatory mediators, and atrial fibrosis is commonly seen in AF. Left ventricular hypertrophy ( $\mathrm{LVH})$ is a common consequence of hypertension, and they are both recognised risk factors for AF. Several studies have noted an association with $\mathrm{LVH}$ and impaired glucose tolerance and insulin resistance. The duration of diabetes and a higher $\mathrm{HbA}_{1 \mathrm{c}}$ are also linked to a greater risk of $\mathrm{AF}{ }^{10}$

Diabetes is additionally well known to have effects on the autonomic nervous system, and several animal studies have demonstrated that diabetic animals were more likely to develop AF after sympathetic nerve stimulation. ${ }^{11}$ The potential mechanisms are summarised in Figure 1.

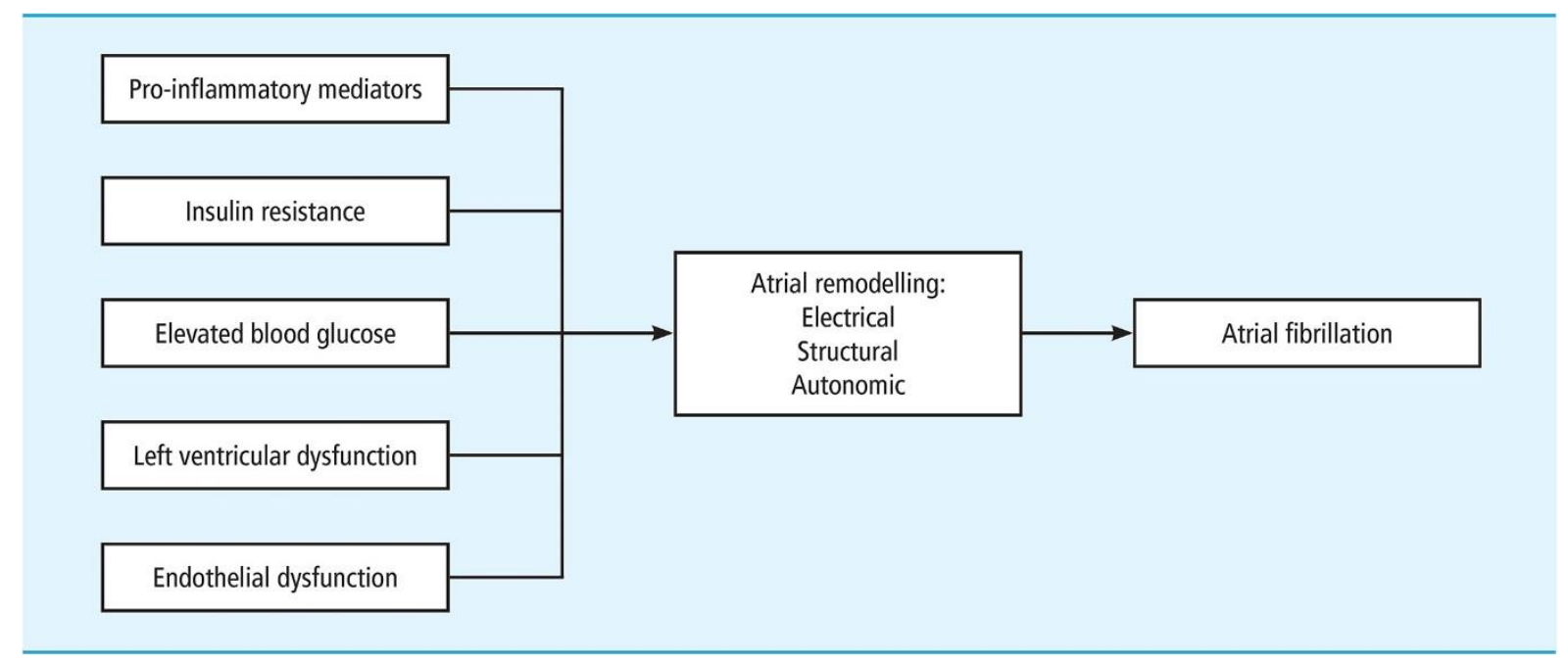

Figure 1 - Potential aetiological links between diabetes and atrial fibrillation

\section{Clinical consequences of atrial fibrillation, and treatment}

Atrial fibrillation can lead to cardiac dysfunction as a result of an uncontrolled heart rate and thromboembolic events due to the irregular rhythm. Therapeutic strategies for rate control include cardioversion to sinus rhythm or using medications to control rate, but the evidence suggests that one approach is no better than the other in terms of prognosis and, in either case, risk stratification and anticoagulation of those deemed to be at risk of thromboembolic events should be normal clinical practice. This also applies to individuals with diabetes. 


\section{Rate versus rhythm control}

A rate control strategy is well-recognised within clinical guidelines. ${ }^{12,13}$ There is little evidence supporting the use of one agent over another in diabetes as most trials assessing their efficacy do not assess outcomes in diabetes and have small numbers.

Traditionally, rate control was achieved using digoxin; however, it has been established that, during exercise or in those with high sympathetic tone, digoxin is less effective. ${ }^{14,15}$ More recently, beta-blockers and non-dihydropyridine calcium channel blockers (NCCBs) have been shown to be more effective than digoxin. The AFFIRM trial also suggested betablockers had a higher success rate for achieving rate control than NCCBs with or without digoxin. ${ }^{16}$ However, this was disputed by another trial, which suggested NCCBs were superior. ${ }^{17}$ The choice of agent is often influenced by the presence of left ventricular dysfunction or a risk of bronchospasm. The use of amiodarone for rate control is largely isolated to the acute setting in unstable patients, and not recommended as a long-term option. There are no trials assessing the efficacy of one rate control strategy over another in those with diabetes.

Rhythm control can be obtained via a variety of methods. Electrical cardioversion is a wellrecognised technique, which has been reported to have a success rate of $90 \%$ in achieving return of sinus rhythm. ${ }^{18}$ However, many will develop AF again within a year. ${ }^{18}$ In one small retrospective study assessing the success of electrical cardioversion in those with diabetes against those without, diabetes and length of time in AF were independent risk factors for failure of electrical cardioversion. ${ }^{19}$ However, this may have been influenced by the presence of greater comorbidity in those with diabetes, as more were dyslipidaemic and hypertensive. Additionally, a raised body mass index (BMI) is known to influence the success of electrical cardioversion. ${ }^{20}$

Chemical cardioversion may be achieved using anti-arrhythmic medications such as amiodarone and flecainide. To the best of our knowledge, there are no studies assessing the efficacy of these drugs in those with diabetes. This may be in part due to the increased risk of arrhythmia in patients with structural heart disease, and the greater prevalence of those with diabetes.

Pulmonary vein isolation and ablation also now play a key role in the management of AF in many patients with paroxysmal and persistent AF which is resistant to conventional antiarrhythmic drug therapy. ${ }^{12}$ There is limited evidence for its use in diabetes, with trials involving small numbers of patients.

A recent review of the evidence $(n=1464)$ suggested that ablation in diabetes was both safe and effective. ${ }^{21}$ Meta-regression analysis found that increased age, BMI and $\mathrm{HbA}_{1 \mathrm{c}}$ were associated with higher rates of recurrence of AF. Additionally, the need for redo ablations was more common in patients with diabetes. There is a suggestion that, due to the greater numbers of comorbidities in patients with diabetes, there is a higher procedural risk associated with ablation in this population. One study found more bleeding events in patients with diabetes when directly compared with those without. ${ }^{22}$ However, a study in Germany assessing periprocedural risks in patients undergoing ablation for $\mathrm{AF}$ and atrial flutter $(\mathrm{n}=$ 8175 ) in patients with and without diabetes found that there was no significant difference 
between the groups in periprocedural or in-hospital complications. ${ }^{23}$ As before, the group with diabetes were older, and had more comorbidities, although they did not have more redo procedures, as previously described. Additionally, a meta-analysis of catheter ablation in those with diabetes found the overall risk of procedural complications to be $3.5 \%$, which was equivalent to the general population. ${ }^{21}$ Longer-term efficacy of ablation for AF in patients with diabetes appears to be comparable with those without diabetes; however, further study is needed to assess both safety and long-term efficacy.

\section{Thromboprophylaxis}

\section{Risk stratification}

There are several risk stratification models available for use to attempt to predict the risk of complications in patients with AF. These include $\mathrm{CHA}_{2} \mathrm{DS}_{2}-\mathrm{VASc}$ and the Framingham schema. ${ }^{2}$ The $\mathrm{CHADS}_{2}$ risk score was previously used in guidelines due to its practicality and relative accuracy. ${ }^{12}$ However, it classifies a large proportion of patients to 'intermediate risk', and has a relatively modest $\mathrm{C}$-statistic score of anywhere from $0.62-0.81$ (over 0.8 being considered a strong model). ${ }^{24,25}$ The Framingham schema is not widely used due to its complexity. More recently, there has been a shift from identifying high-risk patients, to determining which patients are low risk and therefore do not require anticoagulation. This has at least in part been influenced by the availability of a number of alternative oral anticoagulants, and the identification of new and less well-defined risk factors for stroke.

This shift in focus has resulted in the development of the $\mathrm{CHA}_{2} \mathrm{DS}_{2}$-VASc scoring system. ${ }^{26}$ This includes the well-recognised risk factors for stroke including hypertension, diabetes, congestive heart failure and previous stroke, and gives increased weighting to increasing age. It incorporates a weighting for vascular disease, both peripheral and coronary, as well as being female to reflect more recent evidence. This new schema was validated using data from the European Heart Survey, and was found to have a C-statistic of 0.61, second only to the more-complex Framingham study which scored 0.64.

The risk stratification models are shown in Table 1.

Table 1 Risk scores in atrial fibrillation and the maximum points given for each comorbidity

\begin{tabular}{|c|c|c|c|c|c|}
\hline $\mathrm{CHADS}_{2}$ & & $\mathrm{CHA}_{2} \mathrm{DS}_{2}-\mathrm{VASc}$ & & HAS-BLED & \\
\hline Congestive heart failure & 1 & Congestive heart failure & 1 & Hypertension & 1 \\
\hline Hypertension & 1 & Hypertension & 1 & Abnormal liver/renal function & 2 \\
\hline Age $>75$ years & 1 & Age $\geq 75$ years & 2 & Stroke & 1 \\
\hline Diabetes & 1 & Diabetes & 1 & Bleeding & 1 \\
\hline Stroke/TIA & 2 & Vascular disease & 2 & Labile INR & 1 \\
\hline
\end{tabular}




\begin{tabular}{|c|c|c|c|c|c|}
\hline $\mathrm{CHADS}_{2}$ & & $\mathrm{CHA}_{2} \mathrm{DS}_{2}-\mathrm{VASc}$ & & HAS-BLED & \\
\hline- & - & Stroke/TIA & 1 & Age $\geq 65$ years & 1 \\
\hline- & - & Age $65-74$ years & 1 & Drugs/alcohol & 2 \\
\hline- & - & Female & 1 & - & - \\
\hline
\end{tabular}

One of the reasons for further refining the risk stratification in AF is the risk of bleeding with oral anticoagulation. This, in combination with an intermediate risk of stroke, can make decision making regarding anticoagulation challenging for medical practitioners. The HASBLED (hypertension, abnormal liver/renal function, stroke, bleeding history, labile INR, elderly [ $\geq 65$ years], drugs) score was developed to further assist this decision making. When validated, using the European Heart Survey, it gave a C-statistic of 0.72 in the whole population. ${ }^{27}$

There is still some debate about the role of anticoagulation in the group of patients who fall into the $\mathrm{CHA}_{2} \mathrm{DS}_{2}$-VASc score of 1 and the score of 1 non-gender-related score category. A recent meta-analysis of the current evidence found there was a high degree of heterogeneity, and recommended the decision on anticoagulation be made based on individual patient characteristics. ${ }^{28}$ However, in a recent study $(\mathrm{n}=8962)$ patients in AF with a score of 1 nongender-related $\mathrm{CHA}_{2} \mathrm{DS}_{2}$-VASc risk factor were found to have a net clinical benefit when anticoagulated. ${ }^{29}$ The absolute risk at each score is shown in Table 2.

Table 2 Absolute risk associated with risk scores for stroke and bleeding

\begin{tabular}{|lc|cc|}
\hline $\mathbf{C H A}_{2} \mathbf{D S}_{2}$-VASc risk & \multicolumn{2}{|l|}{ HAS-BLED risk } \\
\hline 1 & $1.3 \%$ & 1 & $3.4 \%$ \\
2 & $2.2 \%$ & 2 & $4.1 \%$ \\
3 & $3.2 \%$ & 3 & $5.8 \%$ \\
4 & $4.0 \%$ & & $8.9 \%$ \\
5 & $7.2 \%$ & 4 & $9.1 \%$ \\
6 & & 5 & $>10 \%$ \\
7 & $9.7 \%$ & $>5$ & -
\end{tabular}




\section{Evidence for direct oral anticoagulants}

The management of AF changed with the introduction of direct oral anticoagulant (OAC) medications, and alternative therapies including left-atrial ablation and left-atrial appendage closure. The OAC medications currently licensed for use in AF are dabigatran (direct thrombin inhibitor), apixaban, rivaroxaban and edoxaban (factor Xa inhibitors), and these have begun to replace warfarin (vitamin $\mathrm{K}$ antagonist) in routine clinical practice.

Table 3 summarises the evidence for direct OAC use.

Table 3 Summary of evidence for direct oral anticoagulants

\begin{tabular}{|c|c|c|c|}
\hline \multirow{2}{*}{$\begin{array}{l}\text { Direct oral } \\
\text { anticoagulants }\end{array}$} & \multicolumn{2}{|c|}{ Compared to warfarin } & \multirow{2}{*}{$\begin{array}{l}\text { Percentage with } \\
\text { diabetes }\end{array}$} \\
\hline & $\begin{array}{l}\text { Relative risk of } \\
\text { stroke }\end{array}$ & $\begin{array}{l}\text { Relative risk of } \\
\text { bleeding }\end{array}$ & \\
\hline Dabigatran $110 \mathrm{mg}$ & 0.91 & 0.8 & $23 \%$ \\
\hline Dabigatran $150 \mathrm{mg}$ & 0.66 & 1.16 & $23 \%$ \\
\hline Rivaroxaban & 0.79 & 1.03 & $40 \%$ \\
\hline Apixaban & 0.79 & 0.69 & $25 \%$ \\
\hline Edoxaban $30 \mathrm{mg}$ & 1.07 & 0.47 & $36 \%$ \\
\hline Edoxaban $60 \mathrm{mg}$ & 0.79 & 0.8 & $36 \%$ \\
\hline
\end{tabular}

The RE-LY (Randomised evaluation of long-term anticoagulation therapy) trial ( $\mathrm{n}=18113$ ) was a non-inferiority trial looking at the efficacy and safety of dabigatran at two doses compared to warfarin (target INR 2.0-3.0) ${ }^{30}$ Rates of the primary outcome of stroke and thromboembolism were $1.69 \%$ per year in the warfarin group, as compared with $1.53 \%$ per year in the group that received $110 \mathrm{mg}$ of dabigatran (relative risk with dabigatran $0.91 ; 95 \%$ 
confidence interval [CI] $0.74-1.11 ; \mathrm{p}<0.001$ for non-inferiority) and $1.11 \%$ per year in the group that received $150 \mathrm{mg}$ of dabigatran (relative risk 0.66 ; $95 \%$ CI $0.53-0.82$; $\mathrm{p}<0.001$ for superiority). The rate of major bleeding was $3.36 \%$ per year in the warfarin group, as compared with $2.71 \%$ per year in the group receiving $110 \mathrm{mg}$ of dabigatran $(\mathrm{p}=0.003)$ and $3.11 \%$ per year in the group receiving $150 \mathrm{mg}$ of dabigatran $(\mathrm{p}=0.31)$. Around $23 \%$ of patients in each treatment arm of the study had diabetes. Although the trial achieved its aim by showing non-inferiority to warfarin, there was a trend to better outcomes with both doses of dabigatran.

The ROCKET-AF (Rivaroxaban once daily oral direct factor Xa inhibition compared with vitamin $\mathrm{K}$ antagonism for prevention of stroke and embolism trial in atrial fibrillation) study ( $n=14$ 264) was a double-blind, non-inferiority trial looking at the safety and efficacy of rivaroxaban $20 \mathrm{mg}$ daily versus warfarin. ${ }^{31}$ The primary endpoint of non-inferiority was achieved (hazard ratio in the rivaroxaban group 0.79; 95\% CI 0.66-0.96; $\mathrm{p}<0.001$ for noninferiority). Major and non-major clinically relevant bleeding were similar in each group (hazard ratio $1.03 ; 95 \%$ CI $0.96-1.11 ; \mathrm{p}=0.44$ ), but there was a trend toward less intracranial bleeding in rivaroxaban. Around $40 \%$ of patients had diabetes in the trial, and rivaroxaban was as effective as warfarin in preventing thromboembolic events in these patients, with no difference in the number of bleeding events.

The ARISTOTLE (Apixaban for reduction in stroke and other thromboembolic events in atrial fibrillation) trial $(\mathrm{n}=18$ 201) was a non-inferiority trial looking at the safety and efficacy of apixaban at a dose of $5 \mathrm{mg}$ twice-daily versus warfarin (target INR 2.0-3.0) in patients with $\mathrm{AF}$ and at least one additional risk factor. ${ }^{32}$ In addition to being non-inferior to warfarin (hazard ratio with apixaban $0.79 ; 95 \%$ CI $0.66-0.95 ; \mathrm{p}<0.001$ for non-inferiority), as a secondary endpoint apixaban was superior to warfarin in reducing rates of stroke or systemic embolism $(\mathrm{p}=0.01)$, and the rate of major bleeding was $2.13 \%$ per year in the apixaban group, as compared with 3.09\% per year in the warfarin group (hazard ratio 0.69; 95\% CI $0.60-0.80 ; \mathrm{p}<0.001)$. Around $25 \%$ of patients in ARISTOTLE had diabetes. While the primary outcome was consistent in this subgroup, risk of bleeding was higher compared to those without diabetes mellitus, but with no greater risk than warfarin.

The ENGAGE AF-TIMI 48 trial $(\mathrm{n}=21$ 105) was a non-inferiority trial looking at the efficacy and safety of edoxaban at two doses compared to warfarin (target INR 2.03.0). ${ }^{33}$ Rates of the primary outcome of stroke and thromboembolism were $1.5 \%$ per year in the warfarin group, as compared with $1.18 \%$ per year in the group that received $60 \mathrm{mg}$ edoxaban (hazard ratio $0.79 ; 97.5 \%$ CI $0.63-0.99 ; \mathrm{p}<0.001$ for non-inferiority) and $1.61 \%$ per year in the group that received $30 \mathrm{mg}$ of edoxaban (hazard ratio 1.07 ; $97.5 \%$ CI $0.87-$ $1.31 ; \mathrm{p}=0.005$ for non-inferiority). The rate of major bleeding was $3.43 \%$ per year in the warfarin group, as compared with $2.75 \%$ per year in the group receiving $60 \mathrm{mg}$ of edoxaban (hazard ratio $0.80 ; 95 \%$ CI $0.71-0.91 ; \mathrm{p}<0.001$ ) and $1.61 \%$ per year in the group receiving $30 \mathrm{mg}$ of edoxaban (hazard ratio 0.47 ; $95 \%$ CI $0.41-0.55$; $\mathrm{p}<0.001$ ). Around $36 \%$ of patients in each treatment arm of the study had diabetes with similar results in terms of outcomes. Although the trial achieved its aim by showing non-inferiority to warfarin at both doses, there was a trend to better outcomes with the higher $60 \mathrm{mg}$ dose of edoxaban.

An alternative management option in patients who are unable to be anticoagulated is percutaneous left-atrial appendage closure. This was found in PROTECT AF (Watchman left 
atrial appendage system for embolic protection in patients with atrial fibrillation) which was a non-inferiority trial $(n=707)$ looking at the safety and efficacy of percutaneous catheter leftatrial appendage and closure with filter device (Watchman) versus conventional anticoagulation with warfarin. ${ }^{34}$ It was shown to be non-inferior (relative risk $0.71 ; 95 \% \mathrm{CI}$ 0.44-1.30 per year), which met the criteria for non-inferiority (probability of non-inferiority $>0.999$ ). However, there was no subgroup analysis done on patients with diabetes, and its application in this group of patients requires further study.

\section{Conclusion}

There is a well-recognised relationship between AF and diabetes. However, this relationship is one that remains poorly understood. There are numerous risk stratification scores available, and the $\mathrm{CHA}_{2} \mathrm{DS}_{2}-\mathrm{VASc}$ scoring system is a robust method of categorising low-risk patients. However, there remains a minority group of 'intermediate-risk' patients in whom the decision about whether to anticoagulate is not well-defined.

Anticoagulation with the newer generation of OAC medications is safe and effective. Apixaban was found to have a higher number of bleeding events in patients with diabetes when compared with those without diabetes, but was still comparable with warfarin. There is a similar trend seen in a meta-analysis of three trials (dabigatran, rivaroxaban, apixaban) for those with diabetes, but it did not outweigh the benefits of stroke risk reduction. ${ }^{35}$

Left-atrial ablation and appendage closure offer alternative therapies for the treatment of AF. Ablation has been proven to give good symptomatic and rhythm results; however, in patients with diabetes, further study is required to better define their role in the management of AF.

\section{Declaration of interests}

Prof McKay has done advisory work for Bristol-Myers Squibb and Pfizer.

Prof Fisher and Dr Dobbin have no conflicts of interest to declare.

\section{References}

1. Watson T, et al. Mechanisms of thrombogenesis in atrial fibrillation: Virchow's triad revisited. Lancet 2009;373:155-66.

2. Wang TJ, et al. Temporal relations of atrial fibrillation and congestive heart failure and their joint influence on mortality: the Framingham Heart Study. Circulation 2003;107:2920-5.

3. Zoni-Berisso M, et al. Epidemiology of atrial fibrillation: European perspective. Clin Epidemiol 2014;6:213-20.

4. World Health Organization. Global Report on Diabetes. WHO, 2016. 
5. De Sensi F, et al. Atrial fibrillation in patients with diabetes: molecular mechanisms and therapeutic perspectives. Cardiovasc Diagn Ther 2015;5:364-73.

6. Lin Y, et al. Mechanism of and therapeutic strategy for atrial fibrillation associated with diabetes mellitus. Scientific World J 2013. https://www.hindawi.com/journals/tswj/2013/209428/ [accessed 15 October 2017].

7. Sun Y, Hu D. The link between diabetes and atrial fibrillation: cause or correlation? J Cardiovasc Dis Res 2010;1:10-11.

8. Benjamin EJ, et al. Independent risk factors for atrial fibrillation in a population-based cohort. The Framingham Heart Study. JAMA 1994;271:840-4.

9. Chung MK, et al. C-reactive protein elevation in patients with atrial arrhythmias: inflammatory mechanisms and persistence of atrial fibrillation. Circulation 2001;104:2886-91.

10. Dublin S, et al. Diabetes mellitus, glycaemic control, and risk of atrial fibrillation. J Gen Intern Med 2010;25:853-8.

11. Otake H, et al. Influences of autonomic nervous system on atrial arrhythmogenic substrates and the incidence of atrial fibrillation in diabetic heart. Int Heart $\mathbf{J}$ 2009;50:627-41.

12. NICE. Clinical guidelines CG180. Atrial fibrillation: management. 2014. https://www.nice.org.uk/guidance/cg180 [accessed 15 October 2017].

13. Kirchhof $\mathrm{P}$, et al. 2016 ESC guidelines for the management of atrial fibrillation developed in collaboration with EACTS. Eur Heart J 2016;37:2893-962.

14. Blumgart $\mathrm{H}$. The reaction to exercise of the heart affected by auricular fibrillation. Heart 1924;11:49.

15. Redfors A. Digoxin dosage and ventricular rate at rest and exercise in patients with atrial fibrillation. Acta Med Scand 1971;190:321-33.

16. Olshansky B, et al. The Atrial Fibrillation Follow-up Investigation of Rhythm Management (AFFIRM) study: Approaches to control rate in atrial fibrillation. J Am Coll Cardiol 2004;43:1201-8.

17. Ulimoen SR, et al. Comparison of four single-drug regimens on ventricular rate and arrhythmia-related symptoms in patients with permanent atrial fibrillation. Am J Cardiol 2013;111:225-30.

18. Gall NP, Murgatroyd MA. Electrical cardioversion for AF - the state of the art. Pacing Clin Electrophysiol 2007;30:554-67.

19. Soran $\mathrm{H}$, et al. Influence of diabetes on the maintenance of sinus rhythm after a successful direct current cardioversion in patients with atrial fibrillation. QJM 2008;101:181-7. 
20. Blich M, Edoute Y. Electrical cardioversion for persistent or chronic atrial fibrillation: outcome and clinical factors predicting short and long term success rate. Int J Cardiol 2006;107:389-94.

21. Anselmino M, et al. Catheter ablation of atrial fibrillation in patients with diabetes mellitus: a systematic review and meta-analysis. EP Europace 2015;17:1518-25.

22. Tang RB, et al. Safety and efficacy of catheter ablation of atrial fibrillation in patients with diabetes mellitus - single center experience. J Interv Card Electrophysiol 2006;17:41-6.

23. Bogossian $\mathrm{H}$, et al. Catheter ablation of atrial fibrillation and atrial flutter in patients with diabetes mellitus: who benefits and who does not? Data from the German ablation registry. Int J Cardiol 2016;214:25-30.

24. Wang TJ, et al. A risk score for predicting stroke or death in individuals with new-onset atrial fibrillation in the community: the Framingham Heart Study. JAMA 2003;290:1049-56.

25. Olesen JB, et al. Validation of risk stratification schemes for predicting stroke and thromboembolism in patients with atrial fibrillation: nationwide cohort study. BMJ 2011;342:d124.

26. Lip GY, et al. Refining clinical risk stratification for predicting stroke and thromboembolism in atrial fibrillation using a novel risk factor-based approach: the Euro Heart Survey on atrial fibrillation. Chest 2010;137:263-72.

27. Pisters R, et al. A novel user-friendly score (HAS-BLED) to assess 1-year risk of major bleeding in patients with atrial fibrillation: the Euro Heart Survey. Chest 2010;138:1093100 .

28. Joundi RA, et al.; and on behalf of the Stroke Outcomes Research Working Group. Ischemic stroke risk in patients with atrial fibrillation and CHA2DS2-VASc score of 1. Stroke 2016;47:1364-7.

29. Fauchier L, et al. Should atrial fibrillation patients with only 1 nongender-related CHA2DS2-VASc risk factor be anticoagulated? Stroke 2016;47:1831-6.

30. Connolly SJ, et al. Dabigatran versus warfarin in patients with atrial fibrillation. N Engl J Med 2009;361:1139-51.

31. Patel MR, et al. Rivaroxaban versus warfarin in nonvalvular atrial fibrillation. N Engl J Med 2011;365:883-91.

32. Granger CB, et al. Apixaban versus warfarin in patients with atrial fibrillation. N Engl J Med 2011;365:981-92.

33. Giugliano RP, et al. Edoxaban versus warfarin in patients with atrial fibrillation. N Engl J Med 2013;369:2093-104.

34. Vivek Y, et al. Percutaneous left atrial appendage closure for stroke prophylaxis in patients with atrial fibrillation. Circulation 2013;127:720-9. 
35. Lega J-C, et al. Consistency of safety and efficacy of new oral anticoagulants across subgroups of patients with atrial fibrillation. PLoS One 2014; 9(3): e91398. 